\title{
En búsqueda de una solución óptima al balanceo de mallas curriculares con metaheurísticas y programación funcional
}

\author{
José M. Rubio ${ }^{1}$, Cristian L. Vidal-Silva ${ }^{*}$ y Guillermo Cabrera ${ }^{3}$ \\ (1) Escuela de Computación e Informática, Facultad de Ingeniería, Ciencia y Tecnología, Universidad Bernardo \\ O’Higgins, Avenida Viel 1497, Ruta 5 Sur, Santiago - Chile, (correo-e: josemiguel.rubio@ubo.cl). \\ (2) Departamento de Administración, Facultad de Economía y Administración, Universidad Católica del Norte, Angamos \\ 0610, Antofagasta - Chile, (correo-e: cristian.vidal@ucn.cl). \\ (3) Escuela de Ingeniería Informática, Facultad de Ingeniería, Pontificia Universidad Católica de Valparaíso, Av. Brasil \\ 2241, Valparaíso - Chile, (correo-e: guillermo.cabrera@pucv.cl).
}

* Autor a quien debe ser dirigida la correspondencia.

Recibido May. 27, 2020; Aceptado Jul. 23, 2020; Versión final Ago. 17, 2020, Publicado Dic. 2020

\begin{abstract}
Resumen
El principal objetivo de este trabajo es presentar y ejemplificar una solución computacional para minimizar el costo de diseñar planes curriculares mediante algoritmos bio-inspirados con el fin de automatizar y reducir los errores. Se resuelve el problema de balanceo de mallas curriculares (BACP, por sus siglas en inglés) mediante la metaheurística de optimización basada en el comportamiento o algoritmo de las luciérnagas y el uso de programación funcional en el lenguaje Haskell. Se aplica el algoritmo de las luciérnagas sobre un conjunto de instancias de prueba para demostrar su efectividad. Los resultados muestran que al aplicar la propuesta de funciones solución en los problemas de optimización de carga académica balanceada de ocho, diez y doce semestres, el rendimiento considerando 50 ejecuciones suele ser oscilante, pero permite obtener los valores esperados de mínima carga académica para cada caso. Se concluye que esta propuesta permite la generación eficiente de soluciones para el diseño de planes curriculares.
\end{abstract}

\section{Seeking an optimal solution for a balanced academic curriculum by metaheuristics and functional programming}

\begin{abstract}
The main objective of this study is to present and exemplify a computational solution to minimize the cost of designing curriculum plans by using bio-inspired algorithms to automate and reduce errors. The study focuses on solving the balanced academic curriculum problem (BACP) through metaheuristic optimization, based on the behavior or algorithm of fireflies and based on the use of functional programming in the Haskell language. The firefly algorithm is applied to a set of test instances to demonstrate its effectiveness. The results show that by applying the proposed solution function to optimize load balance in curricular meshes of eight, 10, and 12 semesters, the performance is oscillating when considering 50 runs. But, it permits obtaining expected values for the minimum academic load in each case. It is concluded that the proposal presented here allows generating efficient solutions for designing balanced curricular meshes.
\end{abstract}

Keywords: balancing curricular mesh; metaheuristics; firefly algorithm; functional programming; Haskell 


\section{INTRODUCCIÓN}

Tal como lo describe Rubio et al. (2019), las mallas curriculares no sólo son importantes para diferenciar una misma carrera que se imparta en 2 o más instituciones de educación superior. Estas son un instrumento que contiene los conocimientos y objetivos de aprendizaje que debe alcanzar cada estudiante en una carrera específica, por lo que son diseñadas por docentes, catedráticos y maestros con el fin de satisfacer las necesidades de formación de futuros profesionales, quienes deberían ser capaz de solucionar problemas comunes del área en la que se formaron.

Diseñar una malla curricular es todo un desafío, no sólo porque estas deben formar un profesional integro, sino que además porque esto se debe lograr en un periodo de tiempo determinado y las materias que se deben abordar muchas veces podrían requerir tiempo adicional para su completo desarrollo (Glatthorn et al., 2019). Por estas razones, las mallas curriculares deben ser balanceadas ya que impactan la probabilidad de éxito de un estudiante. Demasiados ramos (cursos o módulos) en un periodo académico podrían ocasionar el fracaso de futuros profesionales, entonces, tal y como señala Rubio et al. (2019), la carga académica debe ser similar en cada periodo y la mínima posible dentro del amplio espectro de competencias y conocimiento que los estudiantes podrían adquirir.

Así, el problema del currículo académico equilibrado (en inglés, Balanced Academic Curriculum Problem BACP) consiste en asignar cursos a períodos de enseñanza que satisfacen los requisitos previos y equilibran la carga de los estudiantes en términos de créditos y número de cursos (Chiarandini et al., 2012). Castro y Manzano (2001) presentaron el BACP junto con proponer y desarrollar un modelo de programación lineal con la consideración de las siguientes entidades y restricciones: 1. Cursos no opcionales (cursos con sus créditos según el currículum académico); 2. Periodos académicos según la malla curricular que se corresponden a un número fijo de intervalos de tiempo (cada periodo académico incluye cursos para enseñar); 3 . Carga académica máxima permitida (número máximo de créditos y cursos para cada periodo académico); 4 . Carga académica mínima permitida (número mínimo de créditos y cursos para cada periodo académico); 5. Prerrequisitos para cada uno de los cursos (algunos cursos deben ser enseñados y aprobado antes que, esto es, son requisitos de otros (los prerrequisitos y los cursos subsecuentes permiten generar pares ordenados de cursos en los que la restricción es que un estudiante debe cursar y aprobar el primer curso antes de cursar el segundo); 6. Distribución equilibrada de la malla curricular, es decir, el número de créditos de cada periodo académico debe ser similar, idealmente igual. Así, el horizonte de planificación BACP se divide en años académicos, los que se dividen en periodos en el que los cursos pueden tener lugar. El problema consiste en encontrar una asignación de cursos a períodos académicos que satisfagan ciertos límites de carga y requisitos previos (Rubio et al., 2018). Este trabajo considera BACP como un problema de optimización.

Algunos trabajos (Hnich y Kiziltan, 2002) definen un modelo estructural y de comportamiento para el problema BACP mientras que otros (Castro y Manzano, 2001) lo tratan como un problema de restricciones. En los últimos años, se han desarrollado diversas propuestas y revisiones de soluciones algorítmicas para problemas con restricciones y de optimización con un enfoque bio-inspirado, usualmente especializaciones u optimizaciones de algoritmos genéticos (Yang 2010; Cabrera et. al, 2012; Kar, 2016; Lanza-Gutiérrez et al., 2017; García et al., 2019; Soto et al., 2019). El Algoritmo de Luciérnaga (en inglés, Firefly Algorithm FA) (Yang 2010) es un ejemplo de dichas soluciones cuya base de funcionamiento son los patrones de parpadeo y comportamiento de las luciérnagas en búsqueda de una solución potencialmente óptima.

Considerando el impacto de una carga académica balanceada para con el desempeño, y éxito académico y profesional de los estudiantes, el objetivo de este trabajo es presentar una solución para minimizar el costo de diseñar planes curriculares balanceados mediante la aplicación de algoritmos bio-inspirados para automatizar y reducir los errores en el diseño de un currículo académico equilibrado (Rubio et al., 2018), es decir, encontrar una asignación de cursos para cada periodo académico los que cumplan con todas las entidades y restricciones mencionados de forma óptima. Este trabajo plantea una solución de optimización de BACP mediante una metaheurística bio-inspirada de optimización basada en el comportamiento algorítmico de atracción de las luciérnagas (Yan, 2010) implementadas en el lenguaje de programación funcional Haskell (Hutton, 2016; Thompson, 2008).

Se aplicará el algoritmo de luciérnaga sobre un conjunto de instancias de prueba obtenidas desde CSPLib (BACP8, BACP10, y BACP12) (Gent et al., 2019), cuyas soluciones se representan en forma de una matriz binaria. Al poseer un conjunto de soluciones válidas, las cuales representan un conjunto de luciérnagas, se procede a optimizar el espacio de soluciones mediante el algoritmo y así poder encontrar una solución óptima. Por ejemplo, en BACP8 hay un currículo compuesto de 46 cursos con un total de 133 créditos a impartir en 8 periodos académicos. En consecuencia, la media aritmética simple de los créditos por cada período académico es equivalente a $133 / 8=16,625$, por lo que 17 representa un potencial límite inferior del número máximo de créditos por período en búsqueda de soluciones óptimas. 


\section{HASKELL Y PROGRAMACIÓN LÓGICA}

Según Hutton (2016), Haskell es un lenguaje de programación multipropósito, funcionalmente puro, y polimórficamente tipificado, que se basa en el cálculo lambda. Como lenguaje de programación funcional, en Haskell, el constructor primario o principal es la función. El lenguaje tiene sus orígenes en las observaciones de Haskell Curry y sus descendientes intelectuales por sus trabajos en lógica matemática (Hughes, 1989).

Los lenguajes de programación orientados a objetos y estructurados son ejemplos de lenguajes de programación imperativos, es decir, lenguajes que definen soluciones algorítmicas como una secuencia de pasos que se ejecutan de manera lógica y estricta en el orden definido. Tal y como resaltan (Hughes, 1989; Hutton, 2016), una de las grandes propiedades de Haskell es, por su naturaleza funcional, permite la definición de funciones y expresiones las que se ejecutan mediante su evaluación. Así, Haskell es un ejemplo de lenguaje de programación de propósito general de alto nivel.

\begin{tabular}{|c|c|}
\hline $\begin{array}{l}\text { fac }:: \text { Int }->\text { Int } \\
\text { fac } 0=1 \\
\text { fac } n \mid n>0=n{ }^{*} \text { fac }(n-1)\end{array}$ & $\begin{array}{l}\text { int fac(int } n)\{ \\
\text { if }(n==0) \\
\text { return } 1 ; \\
\text { else } \\
\text { return } n^{*} \operatorname{fac}(n-1) ;\end{array}$ \\
\hline a. Código Haskell & b. Código Java \\
\hline
\end{tabular}

Fig. 1. Códigos Haskell y Java ejemplos de función factorial.

La figura 1 muestra el código Haskell y Java de la función para, dado un número entero, determinar su número factorial. Nótese que el código Haskell es análogo a la definición o declaración matemática de la función factorial, mientras que el código procedimental de Java requiere indicar paso a paso cada una de las acciones de la función para obtener resultados. A continuación, se detallan propiedades destacables de soluciones Haskell: 1. Brevedad: los programas tienden a ser mucho más breves; 2. Simplicidad: los programas funcionales usualmente son más simples de entender; 3. Sin errores de memoria: como las soluciones son fuertemente tipificadas, no existen incompatibilidad de tipos ni errores de memoria asociados; 4. Reutilización: soluciones Haskell presentan mínimas restricciones para facilitar su reutilización; 5. Alta abstracción: Los lenguajes de programación funcional se basan en la declaración de funciones lo que permite alcanzar altos niveles de abstracción procedimental; esto es, Haskell no se basa en los cambios de estado de variables y objetos como la programación imperativa tradicional; 6 . Administración de memoria: no se requiere una administración explícita de memoria dinámica como en lenguajes de programación imperativa tradicional (Grust, 2014; Hutton, 2016).

Según Hutton (2016), las soluciones funcionales, según las propiedades inherentes de la programación funcional, requieren altos niveles de uso tanto de memoria como de computación, lo cual requería de sistemas de computación de gran nivel en la década anterior. Sin embargo, por los avances y actual disponibilidad y accesibilidad de sistemas de computación de alto rendimiento, las soluciones de programación funcional son mucho más accesibles y viables hoy en día.

\section{ALGORITMO DE LUCIERNAGA Y BACP}

Para aplicar el algoritmo de las luciérnagas (Fireflies Algorithm en inglés, FA) sobre un problema de optimización, es necesario considerar el brillo proporcional al valor de la función objetivo. En el caso de algoritmos genéticos, se puede definir el brillo en el mismo sentido que la función objetivo. Ya que el atractivo o luminosidad de una luciérnaga es proporcional al emanando de luz, la ecuación 1 define esta atracción.

$$
\beta=\beta_{0} \varepsilon^{\lambda r^{2}}
$$

La figura 2 muestra un esquema general de algoritmo de luciérnagas de (Durkita, 2011) cuya función objetivo se define en función del objetivo del problema. Además, es necesario definir la intensidad de luz inicial e inicializar los parámetros de la luciérnaga, el tamaño de la población n y el número máximo de generaciones GeneraciónMax. La idea principal para resolver el BACP propuesto en este trabajo se basa en representar el problema a través de un arreglo binario dada la evidencia de que es muy útil para plantear la solución, además de la adaptación directa del algoritmo de luciérnagas para este fin, tal y como se presenta en la siguiente sección. 


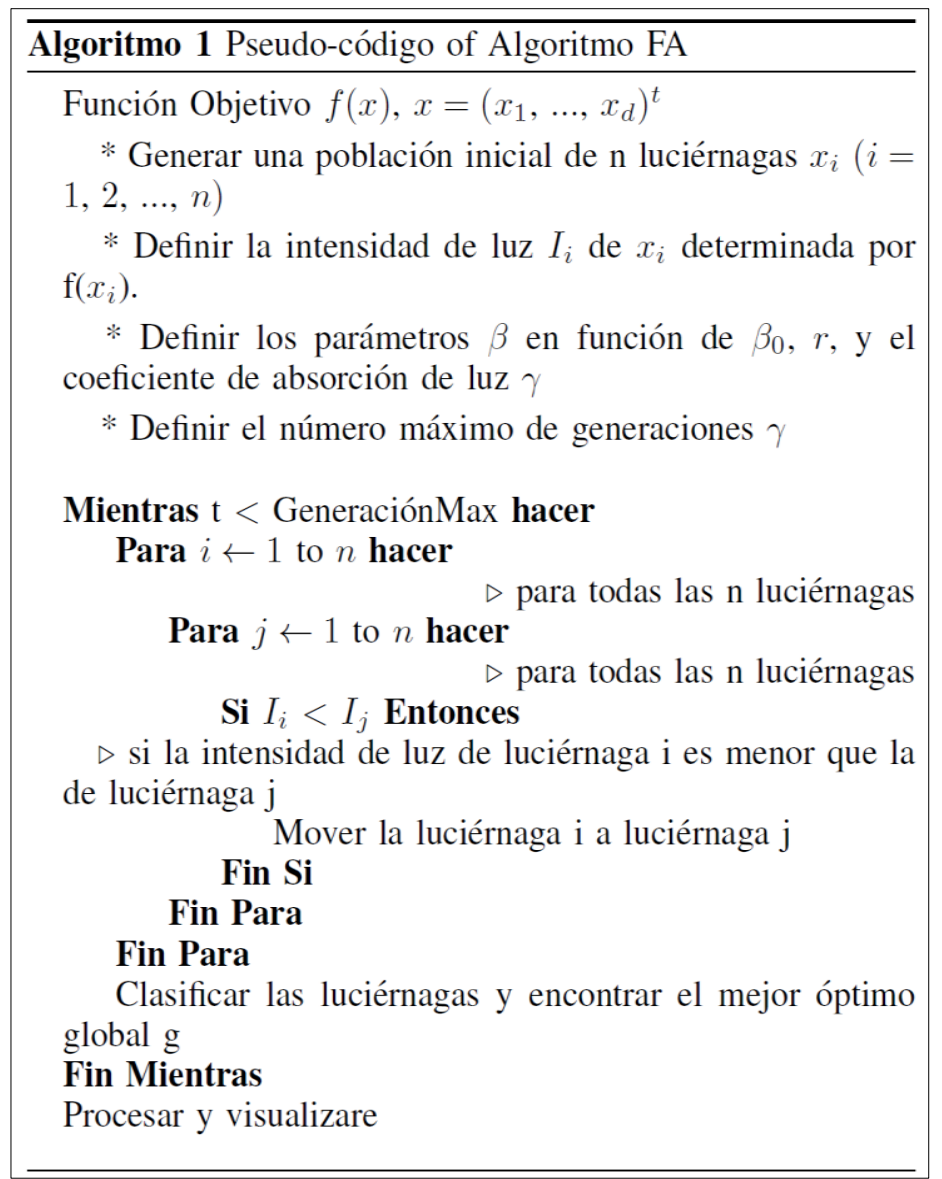

Fig. 2. Algoritmo de las luciérnagas.

\section{METODOLOGÍA}

Como esta investigación consiste en el desarrollo de un algoritmo bio-inspirado para la optimización de mallas curriculares en varias etapas mediante una producción modular, se ha elegido la metodología de desarrollo espiral por su mayor flexibilidad y facilidad para el desarrollo de soluciones incrementales y/o evolutivas de ingeniería de software (Sommerville, 2015), esto es, con la aplicación gradual y evolutiva de etapas de análisis, diseño, implementación y prueba de funcionalidades y módulos definidos. La figura 3 ilustra esta metodología de desarrollo. El modelo espiral incremental, en el contexto de este trabajo, se aplicará en el desarrollo de cuatro fases de prototipado: descubrimiento, codificación, validación y optimización, con una incorporación gradual de restricciones del problema junto con pruebas para determinar la validez del mínimo producto viable en desarrollo y luego en fase de producción.

Para la elección de heurísticas de solución, se utilizó la metodología de diseño planteada por Bird (Bird, 2010) que consiste en el uso de identidades entre funciones para simplificar expresiones algebraicas en programación funcional, y optimizar la implementación de funciones en Haskell utilizando reglas de inferencia para modificar la complejidad de una función y así diseñar una estrategia heurística de reparación global para mejorar la satisfacción de restricciones de precedencias en el BACP. Este trabajo adapta a Haskell los elementos presentados en el trabajo de Rubio et al. (2018). El primer paso fue implementar el modelo matemático original de Castro y Manzano (Castro y Manzano, 2001) en lenguaje Haskell. Para generar un conjunto de soluciones iniciales (población inicial), se consideran la restricción de precedencia y la restricción de carga académica, para luego aplicar optimización mediante el algoritmo de luciérnagas incorporando las demás restricciones del problema.

En el funcionamiento algorítmico de la solución, primero, se lee un grafo de precedencias, y luego elimina los cursos del último periodo para obtener un nuevo grafo. Este proceso se realiza iterativamente hasta que se obtiene un conjunto de cursos sin restricciones de precedencia entre sí (eliminación de precedencias por bloques), para así asegurar que los cursos del conjunto final no tengan conflictos de precedencia. En esta solución, se utilizan funciones que operan sobre listas de la forma $\left(c,\left[p_{1}, . ., p_{n}\right]\right)$ donde $\left[p_{i}\right]$ con $\left.i \in[1, \ldots, n]\right]$ representa la lista de cursos precedentes $\mathrm{p}_{\mathrm{i}}$ al curso $\mathrm{c}$. Por ejemplo, la figura 4 ilustra la estructura de datos que se utiliza para el caso de la instancia BACP8, es decir, para una malla curricular de 8 semestres. 


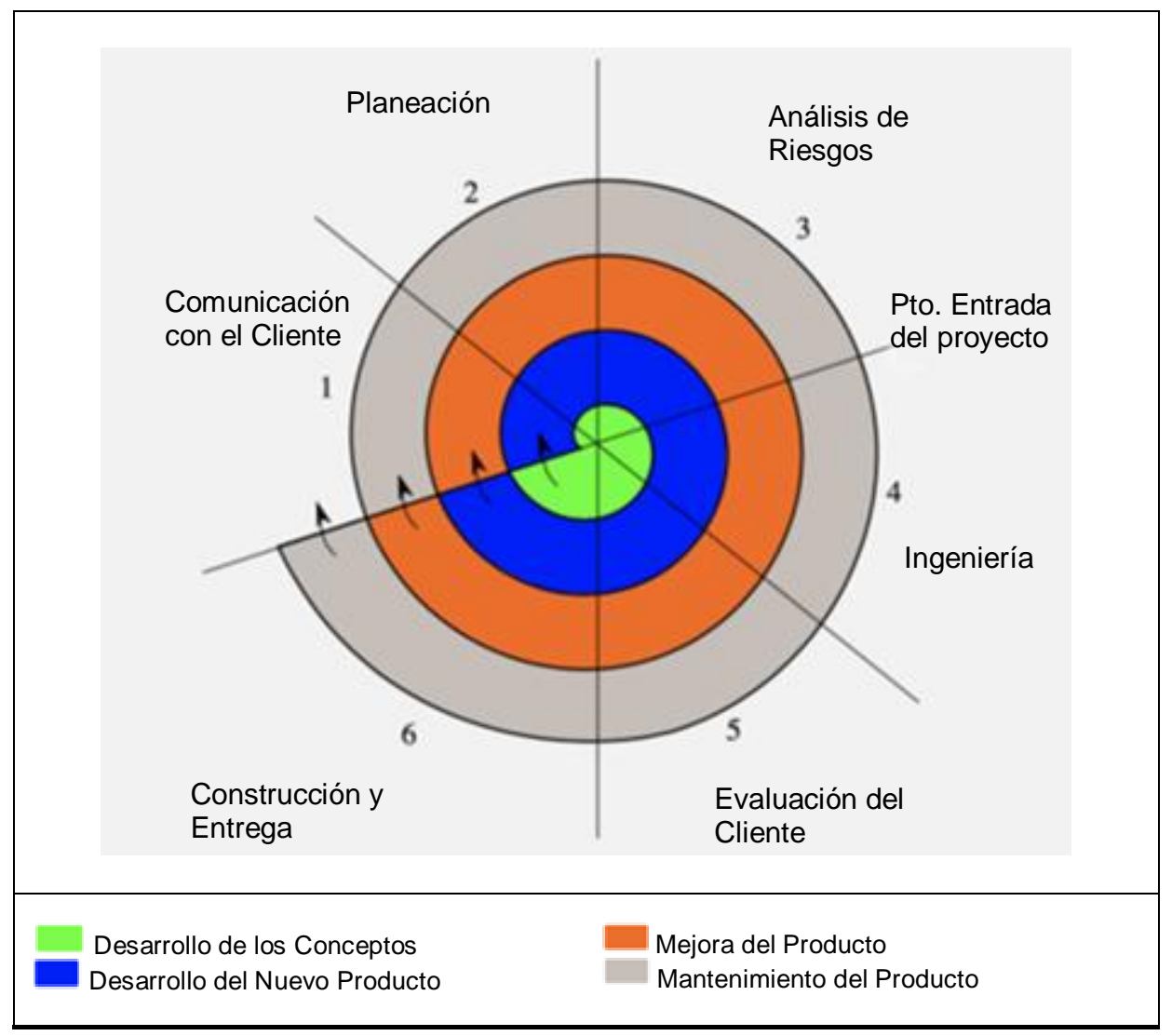

Fig. 3. Metodología espiral simplificada (Unhelkar, 2017).

$$
\begin{aligned}
\text { graphBACP8 }= & (46,[32]),(45,[38]),(40,[36]), \\
& (39,[30]),(38,[36]),(37,[30], \\
& (36,[28]),(35,[28]),(34,[30]), \\
& (33,[26]),(32,[26]),(29,[24]), \\
& (28,[22]),(27,[21]),(26,[14]), \\
& (24,[16]),(21,[15]),(20,[14]), \\
& (19,[14]),(18,[3]),(17,[7]), \\
& (16,[10,11]),(15,[9]),(14,[9]), \\
& (12,[8,11]),(11,[5,6]),(10,[5]), \\
& (8,[2,6]),(7,[1])]
\end{aligned}
$$

Fig. 4. Definición en Haskell de matriz de correlación entre cursos y sus prerrequisitos

La figura 5 presenta parte de la solución Haskell de este trabajo para la generación de nuevos valores en una matriz de BACP, específicamente, para la modificación de valores según algoritmo de luciérnagas de Yang (2010). Nótese que cada definición funcional en la figura 5 presenta comentarios (los comentarios en Haskell se preceden de al menos 2 guiones consecutivos).

\section{RESULTADOS}

Al aplicar la propuesta de funciones solución en los problemas de optimización de carga académica balanceada de ocho, diez y doce semestres, los resultados obtenidos son similares a los obtenidos tanto en los trabajos de Rubio et al. (2018) y Rubio et al. (2019), donde el rendimiento considerando 50 ejecuciones suele ser oscilante, pero permite obtener los valores esperados de mínima carga académica para cada caso. Esto se puede ver en la figura 6 que ilustra los resultados después de 50 ejecuciones de la solución implementada en Haskell para las instancias BACP8, BACP10 y BACP12 respectivamente. Cada ejecución consideró 500 generaciones de luciérnagas (iteraciones). Sin embargo, en todos los casos estudiados el algoritmo converge a la solución óptima muy tempranamente (antes de 50 iteraciones). Por ejemplo, en el caso del BACP8, se comienza con una carga de 18 créditos, para descender aceleradamente luego de dos 
iteraciones hasta la mínima carga de 17. Para el caso de BACP10, se comienza en 16 créditos y tras dos iteraciones, se alcanza el valor de 15, para finalmente, luego de 38 iteraciones, llegar al valor de mínima carga de 14. Finalmente, para BACP12, se comienza en 20 créditos, para luego de dos iteraciones bajar a 19, alcanzando luego de tan sólo diez generaciones, la carga óptima de 18.

La gran ventaja de la solución Haskell es su naturaleza funcional para la definición de declaraciones de lo que se desea obtener como solución, no la secuencia imperativa de pasos con la definición de variables y cambios en sus estados para lograr el objetivo de la solución. Además, tal y como lo ejemplifica (Inca Cheroque, 2012), en la actualidad, las soluciones Haskell incluso presentan rendimiento adecuado y usualmente algunas mejoras respecto a las mismas soluciones en otros lenguajes de programación como C++ y Java.

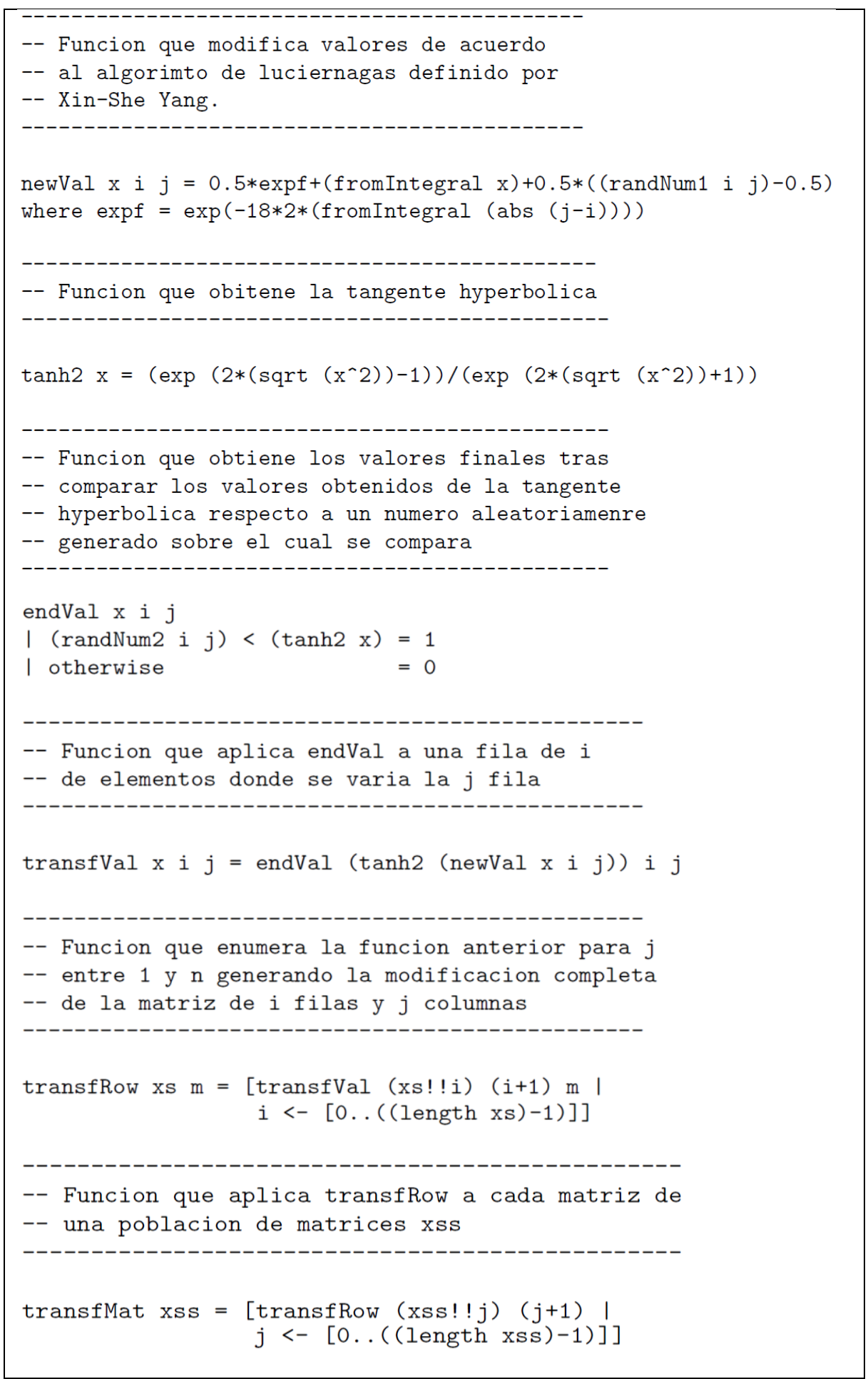

Fig. 5. Funciones Haskell para la generación de nuevos valores en una matriz de correlación de cursos y prerrequisitos. 


\section{DISCUSIÓN}

Dado que el generador de números aleatorios de Haskell es estático y muchos de estos números quedan en memoria una vez generados, su aleatoriedad es aún difícil de controlar, siendo por lo mismo, muy difícil desarrollar un programa totalmente optimizado que resuelva el problema de manera completamente robusta. Cabe destacar que, en futuros desarrollos, se deberá plantear la creación de un tensor de valores aleatorios, que pueda ir modificando iterativamente sus dimensiones dependiendo estas del tamaño de la población, la generación aleatoria y la ejecución de la solución.

Haskell es un lenguaje cuya estructura de programación, tiene el potencial de optimizar muchas tareas relacionadas con el cálculo de elementos extensos en una lista; sin embargo, para poder llegar a un punto donde el tipo de estructura del problema pueda minimizar el tiempo computacional hasta llegar a ser lineal, es necesario trabajar con estructuras de arreglos, de tal manera que estos naturalmente sean llevados a los núcleos de procesamiento mínimo en la máquina desde la que se ejecuta. Justamente, la solución de algoritmo de luciérnagas de este trabajo, con el uso de matrices, busca lograr dicha optimalidad. Como se indica en la sección de Resultados, Haskell permite obtener una solución con un carácter declarativo, para lograr los resultados óptimos, incluso con mayor eficiencia en el desarrollo y rendimiento que soluciones en otros lenguajes de programación tradicional. Este trabajo se implementó como parte de una proyecto de investigación interno de una universidad en Chile.

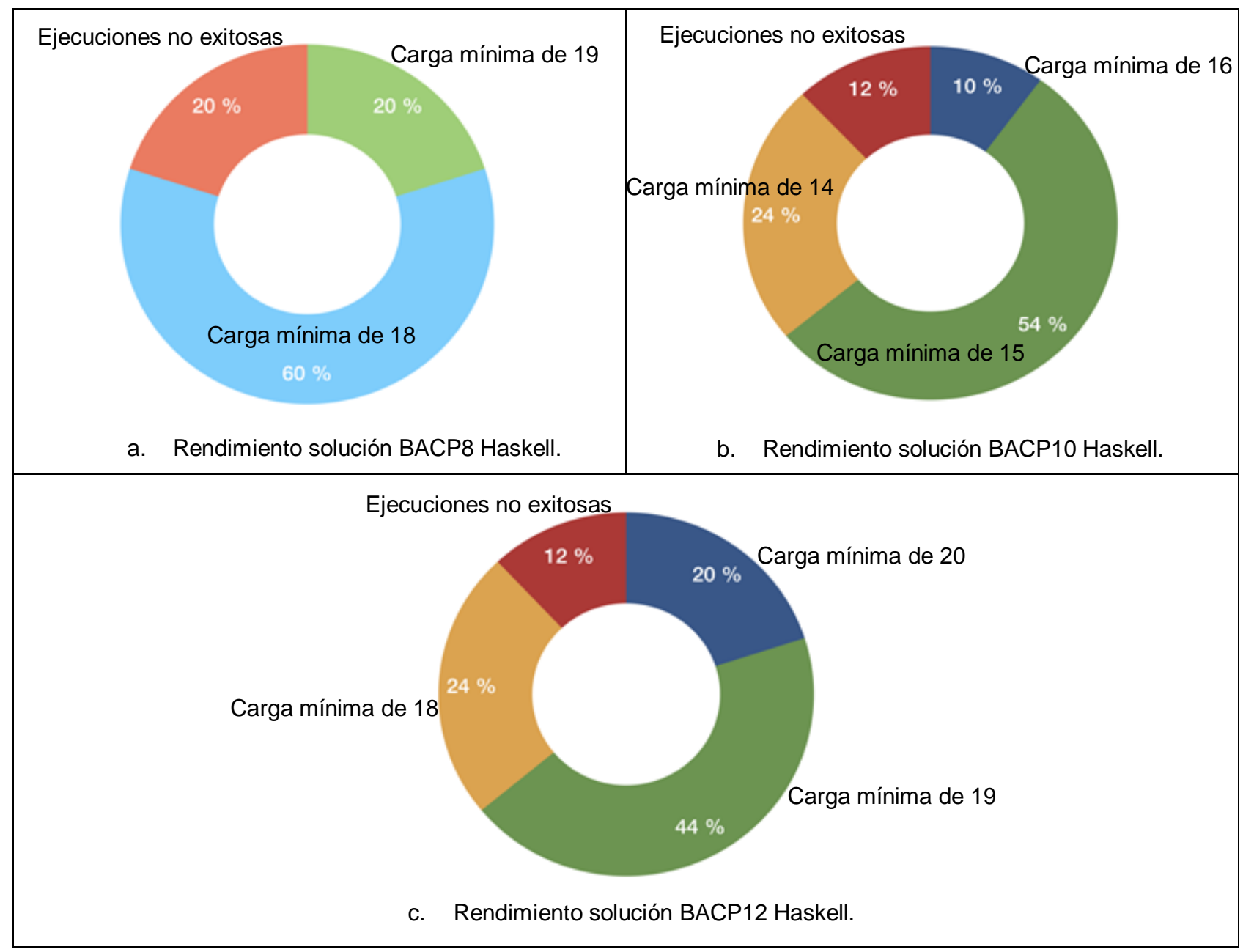

Fig. 6. Rendimiento soluciones BACP8, BACP10 y BACP12 Haskell.

\section{CONCLUSIONES}

Este trabajo presenta una solución algorítmica al problema del BACP en el lenguaje Haskell basada en el paradigma de programación funcional para demostrar la factibilidad teórica y práctica de este enfoque. Así, el mayor aporte de este trabajo es presentar la viabilidad de plantear soluciones funcionales para problemas de optimización con restricciones tales como la generación de una carga académica balanceada para planes de estudio en instituciones de educación superior. Todas las pruebas realizadas demostraron que es posible encontrar soluciones a los casos analizados de la CSPLib. En todos los casos de prueba, la calidad de las 
soluciones es satisfactoria, y permite obtener la solución óptima conocida de cada instancia. Actualmente se está trabajando en demostrar la efectividad de este enfoque para resolver casos reales del problema, y socializar la simplicidad de aplicar soluciones funcionales a problemas de optimización con naturaleza declarativa.

\section{REFERENCIAS}

Bird, R., Pearls of Functional Algorithm Design, ISBN: 0521513383, Cambridge University Press, Ed. 1, New York, NY, USA (2010)

Cabrera, G., Cabrera, E. y otros cuatro autores, A Hybrid Approach Using an Artificial Bee Algorithm with Mixed Integer Programming Applied to a Large-Scale Capacitated Facility Location Problem, doi: 10.1155/2012/954249, Mathematical Problems in Engineering, vol 2012 (954249), 14 (2012)

Castro C. y Manzano, S., Variable and Value Ordering when Solving Balanced Academic Curriculum Problems, Computer Research Repository (CRR), vol. cs, PL/0110007 (2001)

Chiarandini, M., Gaspero, L. D., Gualandi, S. y Schaerf, A., The Balanced Academic Curriculum Problem Revisited, doi: 10.1007/s10732-011-9158-2, Journal of Heuristics, Springer,18 (1), 119-148, Londres, UK (2012)

Durkota, K., Implementation of a Discrete Firefly Algorithm for the Gap Problem within the Sage Framework, in Bachelor Thesis, Czech Technical University, República Checa (2011)

Gent, I. P., Walsh, T. y Selman, B., CSPLib: a Problem Library for Constraints, (en línea), https://www.csplib.org. Acceso: 14 de Marzo (2019)

Glatthorn, A. A., Boschee, F., Whitehead, B. M. y Boschee, B. F., Curriculum Leadership: Strategies for Development and Implementation, ISBN-13: 978-1412992190, SAGE Publications, USA (2019)

García, J., Crawford, B., Soto, R. y Astorga, G., A Clustering Algorithm Applied to the Binarization of Swarm Intelligence Continuous Metaheuristics, doi: 10.1016/j.swevo.2018.08.006, Swarm and Evolutionary Computation, vol. 44, 646 - 664 (2019)

Grust, T., Thinking Functionally with Haskell by Richard Bird, doi:10.1017/S0956796815000076, Journal of Functional Programming, 25 (E2), Cambridge University Press, New York, USA (2014)

Hnich B. y Kiziltan, Z., Modelling a Balanced Academic Curriculum Problem, en Actas de CP-Al-OR-2002, 121 - 131, Nantes, Francia (2002)

Hughes, J., Why Functional Programming Matters, doi: 10.1093/comjnl/32.2.98, The Computer Journal, 32 (2), Oxford University Press, 98 - 107, Oxford, UK (1989)

Hutton, G., Programming in Haskell, ISBN: 1316626229, Cambridge University Press, 2da Ed., New York, USA (2016)

Inca Chiroque, J., Estudio del Lenguaje de Programación Haskell, Ventajas y Desventajas con Respecto a Otros Lenguajes de Programación, Tesis de Magíster en Informática con mención en Ciencias de la Computación, Pontificia Universidad Católica del Perú, Lima, Perú, Septiembre (2012)

Kar, A. K., Bio Inspired Computing - A Review of Algorithms and Scope of Applications, doi: 10.1016/j.eswa.2016.04.018, Expert Systems with Applications: An International Journal, 59(C), 20 - 32, USA, Octubre (2016)

Lanza-Gutiérrez, J. M., Crawford, B. y otros cuatro autores, Analyzing the Effects of Binarization Techniques when Solving the Set Covering Problem through Swarm Optimization. doi: 10.1016/j.eswa.2016.10.05, Expert Systems with Applications, vol. 70, 67 - 82 (2017)

Rubio, J. M., Soto, R. y otros tres autores, Solving the Balanced Academic Curriculum Problem Using Firefly Algorithm, Ingeniare, Revista Chilena de Ingeniería, 26(especial), 102-112, Arica, Chile, Noviembre (2018)

Rubio, J. M., Vidal-Silva, C., Soto, R. y otros tres autores, Applying FireFly Algorithm to Solve the Problem of Balancing Curricula, doi: 10.14569/IJACSA.2019.0100108, International Journal of Advanced Computer Science and Applications, 10(1), UK (2019)

Sommerville, I., Software Engineering, ISBN-13: 978-0133943030, Pearson, Ed. 10, USA, Abril (2015)

Thompson, S., Haskell: The Craft of Functional Programming, ISBN: 0201882957, Addison-Wesley Publishing Company, 3era Ed., USA (2008)

Soto, R., Crawford, B. y otros cuatro autores, Solving the Manufacturing Cell Design Problem through Binary Cat Swarm Optimization with Dynamic Mixture Ratios, doi: 10.1155/2019/4787856, Computational Intelligence and Neuroscience, vol. 44, 1 - 16 (2019)

Tilahun, S. L. y Ngnotchouye, J. M. T., Firefly Algorithm for Discrete Optimization Problems: A Survey, doi: 10.1007/s12205-017-1501-1, KSCE Journal of Civil Engineering, 21(2), 535 - 545, Febrero (2017)

Unhelkar, B, Software Engineering with UML, ISBN: 1138297437, Auerbach Publications, Boston, MA, USA (2017)

Yang, X-S., Firefly algorithm, Levy flights and global optimization, Research and development in intelligent systems XXVI, Springer, 209 - 218, Londres, UK (2010) 